\title{
Concepto de Similitud Geométrica para la Correlación de la Solubilidad Mutua en Mezclas Parcialmente Miscibles de Líquidos lónicos + Alcohol
}

\author{
Luis F. Cardona(1), Claudio A. Faúndez ${ }^{(2)}$ y José O. Valderrama ${ }^{(3,4)}$ \\ 1) Facultad de Ingeniería Química, Departamento de Ingeniería, Grupo de Investigación en Pulpa y Papel, Universidad \\ Pontificia Bolivariana, Circular $1^{\text {a } \# ~ 70-01, ~ M e d e l l i ́ n-C o l o m b i a ~}$ \\ 2) Universidad de Concepción, Depto. de Física, Casilla 160-C, Concepción-Chile \\ 3) Universidad de Antofagasta, Facultad de Ingeniería, Departamento de Ingeniería Química y Procesos de Minerales, \\ Av. Angamos 601, Antofagasta, Chile (e-mail: joseovalderrama@gmail.com) \\ 4) Centro de Información Tecnológica, calle Monseñor Subercaseaux 667, La Serena-Chile
}

Recibido Dic. 14, 2018; Aceptado Feb. 9, 2019; Versión final Feb. 27, 2019, Publicado Jun. 2019

\section{Resumen}

Se desarrolla un modelo de tipo ecuación cúbica de estado para correlacionar la solubilidad de líquidos iónicos en mezclas parcialmente miscibles con alcoholes. El modelo es obtenido a partir del concepto de similitud geométrica entre el diagrama de equilibrio termodinámico presión-volumen-temperatura $(P-v-T)$ y el diagrama temperatura-concentración-presión (T-x-P). Se considera la ecuación de estado de Patel-Teja-Valderrama como el modelo que establece la similitud geométrica y se usan las reglas simples de van der Waals con un parámetro de interacción. Para la estimación de los parámetros del modelo se usan 455 datos experimentales para 28 mezclas binarias (322 datos para la correlación y 133 datos para la predicción). El rango de temperaturas varía entre $277 \mathrm{~K}$ y $403 \mathrm{~K}$, todos a 1 bar de presión. Las desviaciones absolutas obtenidas en la correlación y predicción son similares y menores al 10\%. El modelo es comparado con diferentes enfoques propuestos en la literatura y los resultados muestran que el modelo desarrollado es simple y entrega resultados aceptables para este tipo de mezclas.

\section{The Geometric Similitude Concept for the Correlation of Mutual Solubilities in Partially Miscible Mixtures of lonic Liquids + Alcohols}

\begin{abstract}
A cubic equation of the state model to correlate the solubility of ionic liquids in partially miscible mixtures with alcohols is presented. The model is obtained using the geometric similitude concept between the diagram pressure-volume-temperature $(\mathrm{P}-\mathrm{v}-\mathrm{T})$ at saturation conditions and the solubility diagram pressureconcentration-temperature $(\mathrm{P}-\mathrm{x}-\mathrm{T})$. The model that provides the geometric similitude is the Patel-TejaValderrama equation of state including the simple mixing rules proposed by van der Waals with one interaction parameter. The adjustable parameter is estimated by fitting 455 experimental data for 28 binary mixtures (322 data for correlation and 133 data for prediction). The temperature range varies from $277 \mathrm{~K}$ to $403 \mathrm{~K}$, with all data at 1 bar. Similar deviations below $10 \%$ are obtained for the correlation and prediction steps. The model is compared with different approaches found in the literature and the results show that the proposed model is simpler and provides acceptable results.
\end{abstract}

Keywords: solubility; ionic liquids; cubic equation of state, consolute temperature 


\section{INTRODUCCIÓN}

Los líquidos iónicos (LIs) son sustancias formadas solo por iones y que a temperatura ambiente están en estado líquido. Los LIs han sido llamados solventes "verdes" por su muy baja volatilidad y sus extraordinarias características que pueden ser manejadas en el laboratorio (Shamshina et al., 2015; Hekayati et al., 2015). Por esta particularidad de ser sintetizados de acuerdo a las características que se desea tener de la sustancia, han sido llamados también solventes a medida. Esta amplia posibilidad de manejo de las propiedades que pueden tener los Lls aumenta en forma extraordinaria si se considera mezclas de líquidos iónicos, ya sea entre ellos o con otros solventes orgánicos (Wang et al., 2017). Al igual que muchas sustancias orgánicas los Lls pueden formar mezclas completamente miscibles, parcialmente miscibles o inmiscibles, por lo que estudios de equilibrio líquido-líquido son de especial interés (Wang et al., 2017).

La miscibilidad parcial se observa no sólo en mezclas binarias sino también en sistemas ternarios (y superiores), haciendo así la extracción una posible operación de separación de amplia aplicación (Henley y Seader, 2000). A temperatura y presión atmosférica es relativamente simple obtener en forma experimental las concentraciones de las fases líquidas coexistentes en estos casos de miscibilidad parcial. La literatura técnica y científica es abundante en datos experimentales de solubilidad en mezclas líquidas, pero hay menos datos en el área de los líquidos iónicos (Bai et al., 2011). A presiones más altas, sin embargo, los datos de solubilidad son más escasos. La miscibilidad parcial en los líquidos a menudo se denomina separación de fases, porque efectivamente se forma más de una fase, en el mismo estado líquido. Los criterios termodinámicos que determinan la separación de fases corresponden a los criterios usuales de la termodinámica del equilibrio (Poling et al., 2001, Henley y Seader, 2000; van Ness y Abbott, 1982).

A una presión determinada (fija) la separación de fases puede ocurrir de varias formas a distintas temperaturas, dependiendo del tipo de sustancias mezcladas. Existe así una temperatura característica denominada "consoluta", que limita en distintas formas las zonas de miscibilidad y las zonas parcialmente miscibles: la temperatura consoluta superior Tu y la temperatura consoluta inferior TL. Algunas de estos posibles comportamientos se muestran en la Figura 1. La curva tipo 1 muestra un caso con Tu solamente, indicando que sobre esa temperatura los componentes son completamente miscibles y bajo esa temperatura se forma dos fases con las concentraciones indicadas por la curva de solubilidad. La curva tipo 2 representa una mezcla con $T_{L}$ solamente, indicando que bajo esa temperatura los componentes son completamente miscibles y sobre esa temperatura se forma dos fases con las concentraciones indicadas por la curva de solubilidad. La curva tipo 3 en la Figura 1, representa una mezcla que posee temperatura consoluta superior (Tu) y temperatura consoluta inferior $\left(\mathrm{T}_{\mathrm{L}}\right)$. Entre estas dos temperaturas se forman dos fases con las concentraciones determinadas por la curva de solubilidad.

De los tipos de diagramas mostrados en la Figura 1, los más comunes son los que presentan temperatura consoluta superior (Tipo 1 de la Figura 1). El objetivo de este trabajo es la correlación del equilibrio líquidolíquido de mezclas parcialmente miscibles cuyos datos experimentales siguen el comportamiento Tipo 1 mostrado en la Figura 1. Diferentes mezclas de líquidos parcialmente miscibles se han estudiado y modelado en la literatura (Poling et al., 2001), principalmente usando modelos empíricos, semiteóricos tales como los modelos de Margules, van Laar, Wilson, NRTL, y UNIQUAC (Henley y Seader, 2000).
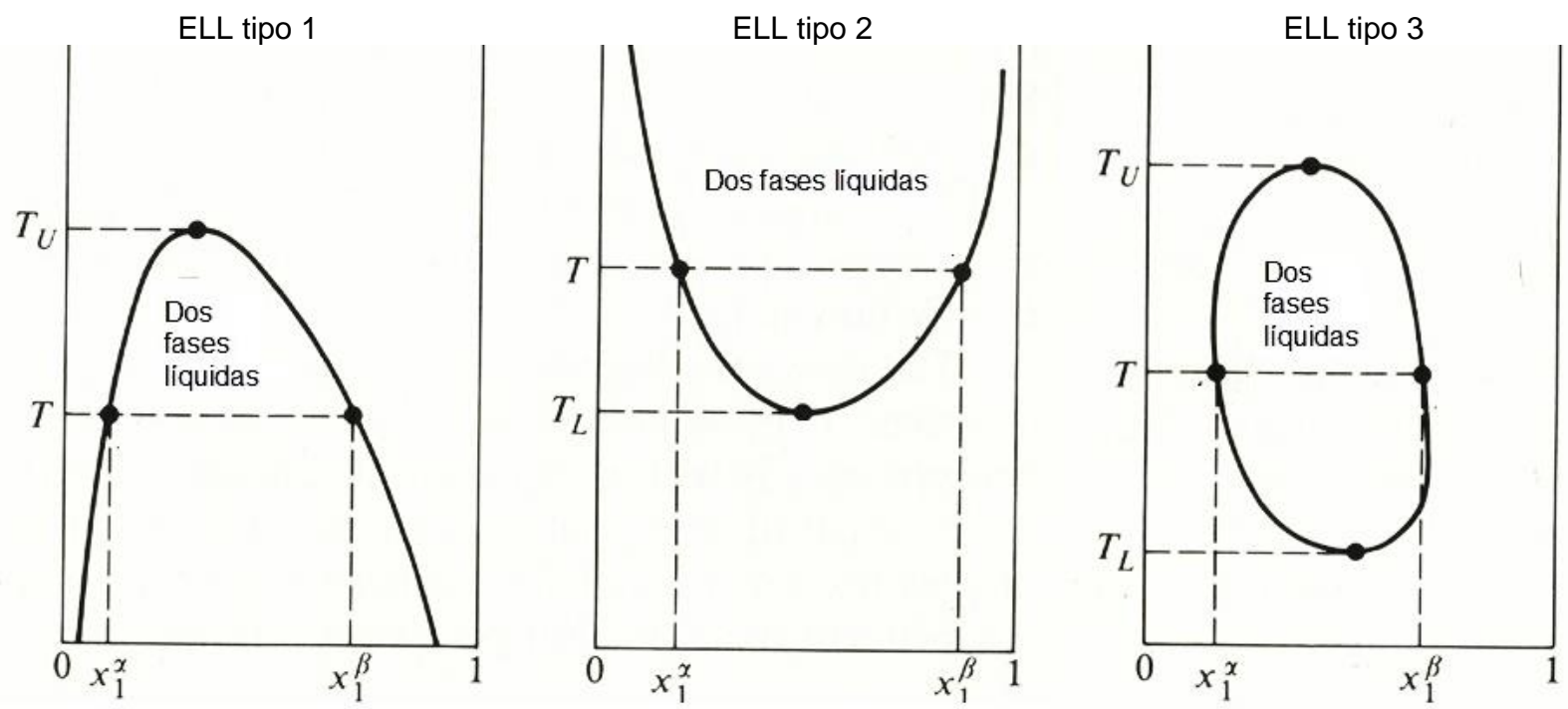

Fig. 1: Tres tipos de comportamiento de ELL en mezclas binarias (Adaptado de van Ness y Abbott, 1982) 
Una alternativa para correlacionar y modelar el comportamiento de propiedades de fluidos y mezclas se ha explorado recientemente por Valderrama et al. (2019a) en otras aplicaciones y es conocida como similitud geométrica. Un método simple que establece que, si dos diagramas cartesianos de variables distintas tienen visualmente la misma forma, la expresión matemática que representa uno de los diagramas debe ser la misma que representa el segundo diagrama. En la Figura 1 la mezcla de tipo 2 (a las que corresponden las mezclas de interés en este trabajo) presentan una curva tipo campana de saturación similar a la campana de saturación encontrada en un diagrama presión-volumen (PV), como se explica en lo que sigue.

Si se hace un gráfico cartesiano de la campana de saturación líquido-vapor de una sustancia se obtiene una figura como la Figura (2a). Si se hace un gráfico cartesiano de la curva de solubilidad Tx, temperatura vs concentración, se obtiene una Figura como la (2b). Se puede observar que la forma de las curvas es similar, pudiéndose argumentar, bajo el concepto de similitud geométrica, que si el primer diagrama (Pv) es geométricamente similar al segundo diagrama (Tx), el modelo matemático que representa el primer diagrama Pv debe ser el mismo modelo matemático que representa el segundo diagrama Tx (Valderrama et al., 2019a). Por ejemplo, si se usa la ecuación de van der Waals (Valderrama, 2003) para representar la curva de saturación líquido-vapor, se escribe: $\mathrm{P}=\mathrm{RT} /(\mathrm{v}-\mathrm{b})-\mathrm{a} / \mathrm{v}^{2}$ y en forma similar la curva Tx podría ser representada por la misma ecuación cambiando las variables ( $v$ por $x)$ : $P=R T /(x-b)-a / x^{2}$.
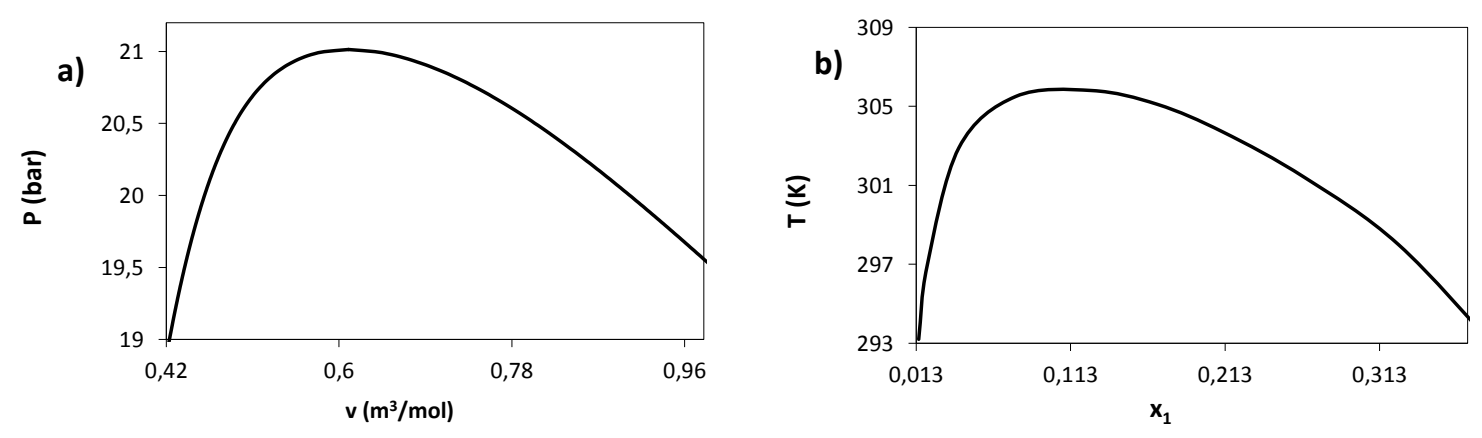

Fig. 2: Comparación entre el diagrama PvT y PxT.

La aplicación del concepto de similitud se ha realizado principalmente en la estimación de las propiedades de transporte, en particular la viscosidad. Este concepto de similitud geométrica fue inicialmente descrito por Philips (1912), mientras estudiaba y analizaba el comportamiento de la viscosidad del dióxido de carbono. A partir del comportamiento de los datos de viscosidad Philips observó la similitud geométrica visual entre el diagrama $\mathrm{P} \rho$ (densidad)T y el diagrama $T \mu$ (viscosidad) $\mathrm{P}$. Durante varios años este concepto no recibió mucha atención hasta que diferentes aplicaciones en la industria de petróleo hicieron revivir de nuevo este concepto. Son Little y Kennedy (1968) quienes desarrollan el primer modelo del tipo ecuación de estado (EdE) a partir de la analogía entre el diagrama $\mathrm{Pv}$ (volumen molar) $\mathrm{T}$ y $\mathrm{T} \mu$ (viscosidad) $\mathrm{P}$. Posteriormente Lawal (1986) propone un modelo de viscosidad a partir de la ecuación de estado de Lawal-Lake-Silberberg, usando la misma analogía entre el diagrama PvT y T $\mu$ P.

Durante los últimos 20 años se han presentado varios modelos usando ecuaciones de estado y el concepto de similitud geométrica. Se han usado las ecuaciones cúbicas de Soave-Redlich-Kwong, Peng-Robinson, y Patel-Teja (Guo et al., 2001; Fan y Wang, 2006; Khosharay et al., 2018; Cardona et al., 2019). Más recientemente, el concepto de similitud geométrica ha sido aplicado por Valderrama et al. (2019b) para correlacionar y predecir la viscosidad de líquidos iónicos puros y de mezclas completamente miscibles de líquidos iónicos. En este trabajo se hace una aplicación distinta del concepto de similitud geométrica, con el fin de correlacionar la solubilidad mutua de líquidos iónicos y alcoholes en mezclas parcialmente miscibles de estas sustancias. En este caso se usa el concepto de similitud geométrica entre la línea de saturación en el diagrama $\mathrm{Pv}$ de una sustancia cualquiera y la línea de saturación en el diagrama Tx de una mezcla parcialmente miscible.

\section{MODELO DE ECUACIÓN DE ESTADO}

Una de las principales ventajas de las ecuaciones cúbicas de estado radica en que para la gran mayoría de aplicaciones prácticas sus parámetros pueden ser ajustados fácilmente para obtener modelos de predicción aplicables a una gran cantidad de sustancias (Valderrama, 2003). Una ecuación cúbica de tres parámetros propuesta por Valderrama (1990) es considerada como uno de los mejores modelos generalizados en la predicción de sustancias no polares y polares (Danesh et al. 1991; Danesh, 1998) y es la seleccionada en este estudio para aplicar el concepto de similitud geométrica. La EdE de Patel-Teja-Valderrama (1990), originalmente propuesta en términos de volumen molar $(\mathrm{v})$, presión $(\mathrm{P})$ y temperatura $(\mathrm{T})$ se define de acuerdo con la Tabla 1. 
Tabla 1: Modelo matemático de la ecuación cúbica de Patel-Teja-Valderrama (1990).

\begin{tabular}{|l|l|}
\hline Descripción & Expresiones matemáticas \\
\hline Forma explícita de la EdE & $P=\frac{R T}{v_{m}-b_{m}}-\frac{a_{m}}{v_{m}\left(v_{m}+b_{m}\right)+c_{m}\left(v_{m}-b_{m}\right)}$ \\
\hline $\begin{array}{l}\text { Parámetros de la EdE del } \\
\text { componente puro }\end{array}$ & $\begin{array}{l}a_{c i}=\left(\phi_{0}+\phi_{1} Z_{c i}\right) \frac{R^{2} T_{c i}^{2}}{P_{c i}} ; a_{i}=a_{c i} \alpha_{i}(T) \quad i=1,2 \\
b_{i}=\left(\beta_{0}+\beta_{1} Z_{c i}\right) \frac{R T_{c i}}{P_{c i}} \quad c_{i}=\left(\gamma_{0}+\gamma_{1} Z_{c i}\right) \frac{R T_{c i}}{P_{c i}} \\
\alpha(T)=\left[1+\mathrm{F}\left(1-(\mathrm{T} / \mathrm{Tc})^{0.5}\right)\right]^{2} \\
\mathrm{~F}=0.4628+3.5823\left(\omega Z_{\mathrm{c}}\right)+8.1942\left(\omega Z_{\mathrm{c}}\right)^{2}\end{array}$ \\
\hline $\begin{array}{l}\text { Regla de mezcla de van der } \\
\text { Waals para mezclas binarias }\end{array}$ & $\begin{array}{l}a_{m}=x_{1}^{2} \cdot a_{1}+2 x_{1} \cdot x_{2} \sqrt{a_{1} \cdot a_{2}} \cdot\left(1-k_{12}\right)+x_{2}^{2} \cdot a_{2} \\
b_{m}=x_{1} \cdot b_{1}+x_{2} \cdot b_{2} \quad c_{m}=x_{1} \cdot c_{1}+x_{2} \cdot c_{2}\end{array}$ \\
\hline
\end{tabular}

En la Tabla 1, aci, bi y $c_{i}($ con $i=1,2)$ son parámetros de cada sustancia pura 1 o 2; $\alpha(T)$ es una función dependiente de la temperatura para expresar la dependencia de la temperatura del parámetro de fuerza "a" en ecuaciones de estado tipo vdW; $R$ es la constante universal del gas ideal; $T$ y $P$ son la temperatura y presión, $T_{c}$ es la temperatura crítica en kelvin, $\mathrm{P}_{c}$ es la presión crítica en bar, $\mathrm{Z}_{\mathrm{c}}$ es el factor de compresibilidad crítico (adimensional); y $\omega$ es el factor acéntrico (adimensional). Diferentes funciones $\alpha(T)$ han sido propuestas en la literatura, cada una con una expresión matemática diferente (Valderrama, 2003). La función alfa descrita en la Tabla 1 corresponde a una de las más usadas en la literatura y fue propuesta por Soave (1972). Además, en la Tabla 1 (fila 4) los términos $\mathrm{am}_{\mathrm{m}}, \mathrm{b}_{\mathrm{m}}, \mathrm{C}_{\mathrm{m}}$ son los parámetros de la mezcla y son estimados usando reglas de mezcla y reglas de combinación. Por su simplicidad y por los diferentes usos en aplicaciones de la industria de procesos la regla de mezcla simple de van der Waals es incorporada en el modelo en estudio. En esta regla de mezcla (Tabla 2, fila 4), $\mathrm{k}_{12}$ es conocido como parámetro de interacción binario. El parámetro de interacción $\mathrm{k}_{12}$ se calcula mediante el ajuste del modelo a datos experimentales de solubilidad.

Aplicando el concepto de similitud geométrica entre los diagramas Pv y Tx (ver Figura 2), se asume que el modelo matemático que describe la relación entre las variables v(volumen molar) $\mathrm{PT}(\mathrm{P}=\mathrm{P}(\mathrm{v}, \mathrm{T}))$ se puede extender para describir la solubilidad de mezclas de líquidos iónicos en alcoholes, temperatura-vs- $\mathrm{X}_{1}$ (fracción molar de líquido), realizando un cambio en las variables v(volumen molar) por $x_{1}$ (fracción molar). El modelo de solubilidad propuesto se muestra en la ecuación 1:

$$
P=\frac{R^{*} T}{x_{1, m}-b_{m}}-\frac{a_{m}}{x_{1, m}\left(x_{1, m}+b_{m}\right)+c_{m}\left(x_{1, m}-b_{m}\right)}
$$

En la ecuación 1, P es la presión en bar, T es la temperatura en kelvin, $x_{1}$ es la fracción molar de líquido iónico (adimensional) y los parámetros de la mezcla $\left(\mathrm{a}_{\mathrm{m}}, \mathrm{b}_{\mathrm{m}}\right.$ y $\left.\mathrm{c}_{\mathrm{m}}\right)$ se determinan mediante las siguientes reglas de mezcla simple del tipo van der Waals de igual manera que el modelo presentado en la Tabla 1:

$$
\begin{aligned}
& a_{m}=x_{1}^{2} \cdot a_{1}-2 x_{1} \cdot x_{2} \sqrt{a_{1} \cdot a_{2}}\left(1-k_{12}\right)+x_{2}^{2} \cdot a_{2} \\
& b_{m}=x_{1} \cdot b_{1}+x_{2} \cdot b_{2} \\
& c_{m}=x_{1} \cdot c_{1}+x_{2} \cdot c_{2}
\end{aligned}
$$

En las ecuaciones 1 a 4, x1 es la fracción molar de líquido iónico (adimensional), x2 es la fracción molar de alcohol (adimensional) y $a_{i}$, bi y $c_{i}$ (con $\left.i=1,2\right)$, son parámetros característicos de cada sustancia (1 el líquido iónico y 2 el alcohol) y se calculan con las mismas ecuaciones reportadas en la Tabla 1, pero los valores de las contantes $\left(R^{*}, \phi_{0}, \phi_{1}, \beta_{0}, \beta_{1}, \gamma_{0}, \gamma_{1}, h_{1}, h_{2}, h_{3}\right)$ son diferentes a los de la ecuación PTv de la Tabla 1.

$$
\begin{aligned}
& a_{i}=a_{c i} \cdot \alpha_{i}(T) \\
& a_{c i}=\left(\phi_{0}+\phi_{1} Z_{c i}\right) \frac{R^{* 2} T_{c i}^{2}}{P_{c i}} \\
& b_{i}=\left(\theta_{0}+\theta_{1} Z_{c i}\right) \frac{R^{*} T_{c i}}{P_{c i}}
\end{aligned}
$$




$$
\begin{aligned}
& c_{i}=\left(\gamma_{0}+\gamma_{1} Z_{c i}\right) \frac{R^{*} T_{c i}}{P_{c i}} \\
& \alpha_{\mathrm{i}}(\mathrm{T})=\left[1+\mathrm{F}_{\mathrm{i}}\left(1-\left(\mathrm{T} / \mathrm{T}_{\mathrm{ci}}\right)^{0.5}\right)\right]^{2} \\
& \mathrm{~F}_{\mathrm{i}}=\mathrm{h}_{1}+\mathrm{h}_{2}\left(\omega_{\mathrm{i}} \cdot \mathrm{Z}_{\mathrm{c}, \mathrm{i}}\right)+\mathrm{h}_{3}\left(\omega_{\mathrm{i}} \cdot \mathrm{Z}_{\mathrm{c}, \mathrm{i}}\right)^{2}
\end{aligned}
$$

En las ecuaciones 5 a 10 que describen el modelo que representa las variables TxP se debe observar lo siguiente: 1) $T_{c}, P_{c}$ y $Z_{c}$ son las propiedades críticas, $T_{c}$ en kelvin, $P_{c}$ en bar y $Z_{c}$ adimensional, $\omega$ es el factor acéntrico (adimensional); 2) el parámetro $\mathrm{R}^{*}$ que reemplaza la constante de los gases ideales que aparece en el modelo PvT (ver Tabla 1) es constante para todas las mezclas analizadas y diferente a la constante de los gases; 3 ) los términos $\phi_{0}, \phi_{1}, \beta_{0}, \beta_{1}, \gamma_{0}, \gamma_{1}$ definidos en la ecuación de estado y relacionados con los parámetros $\mathrm{am}_{\mathrm{m}} \mathrm{b}_{\mathrm{m}}$ y $\mathrm{cm}$, son parámetros que varían dependiendo de la concentración de los componentes en la mezcla; 4) los términos $h_{1}, h_{2}$ y $h_{3}$ relacionados con la función alfa de Soave (1972), varían para cada mezcla; 5) el modelo tiene características empíricas y los parámetros de la ecuación de estado de solubilidad no presentan un sentido físico-químico como lo es la ecuación de estado en volumen PvT; y 6) el modelo es consistente en unidades, si se interpreta en la forma correcta. La Tabla 2 resume las unidades de las variables y parámetros del modelo de EdE usado para relacionar la temperatura con la solubilidad.

Tabla 2: Modelo y unidades de cada uno de los términos del modelo de EdE para la solubilidad

\begin{tabular}{|c|c|}
\hline \multicolumn{2}{|c|}{ Modelo matemático de solubilidad } \\
\hline$P=\frac{R^{*} T}{x_{1}-b_{m}}-\frac{a_{m}}{\left(x_{1}+b_{m}\right)+c_{m}\left(x_{1}-b_{m}\right)}$ en $[\mathrm{bar}] \Leftrightarrow\left\{\begin{array}{l}\frac{R^{*} T}{x_{1}-b_{m}} \text { en }[\mathrm{bar}] \\
\frac{a_{m}}{\left(x_{1}+b_{m}\right)+c_{m}\left(x_{1}-b_{m}\right)} \text { en }[\mathrm{bar}]\end{array}\right.$ \\
\hline Parámetros & Unidades \\
\hline $\mathrm{R}^{*}$ & {$[\mathrm{bar} / \mathrm{K}]$} \\
\hline $\mathrm{a}_{\mathrm{c}, \mathrm{i}}, \mathrm{a}_{\mathrm{m}}$ & {$[\mathrm{bar}]$} \\
\hline $\mathrm{b}_{\mathrm{i}}, \mathrm{C}_{\mathrm{i}}, \mathrm{b}_{\mathrm{m}}, \mathrm{C}_{\mathrm{m}}$ & adimensional \\
\hline $\mathrm{T}, \mathrm{T}_{\mathrm{c}}$ & {$[\mathrm{K}]$} \\
\hline $\mathrm{P}, \mathrm{P}_{\mathrm{c}}$ & {$[\mathrm{bar}]$} \\
\hline $\mathrm{x}_{1}, \mathrm{x}_{2}$ & adimensional \\
\hline$\alpha(\mathrm{T}), \mathrm{Z}_{\mathrm{c}, \omega} \omega, \phi, \phi 1, \beta_{0}, \beta_{1}, \gamma_{0}, \gamma_{1}, \mathrm{~h}_{1}, \mathrm{~h}_{2}, \mathrm{~h}_{3}$ & adimensional \\
\hline
\end{tabular}

\section{DETERMINACIÓN DE PARÁMETROS}

La correlación empírica descrita por las ecuaciones 1-10, presenta 10 parámetros ( $\phi_{0}, \phi_{1}, \beta_{0}, \beta_{1}, \gamma_{0}, \gamma_{1}, h_{1}, h_{2}, h_{3}$ y $R^{*}$ ) que deben ser ajustados utilizando datos experimentales. Los datos experimentales usados son recolectados de la literatura y corresponden a condiciones isobáricas a 1 bar de presión. En total 28 mezclas binarias de $\mathrm{LI}$ en alcohol son consideradas en el estudio, y son resumidas en la Tabla 3 . En esta Tabla, $\mathrm{N}$ es el número de datos experimentales, $\mathrm{T}_{\text {mín }} \mathrm{y} \mathrm{T}_{\text {máx }}$ corresponden a la menor y mayor temperatura y $\mathrm{x}_{1, \text { mín }}$ y $\mathrm{x}_{1 \text {,máx }}$ corresponde a la menor y mayor fracción molar de líquido iónico. Los datos son de (1) Bai et al. (2011), (2) Huo et al. (2008), (3) Domańska y Marciniak (2004) y (4) Crosthwaite et al. (2006).

El modelo desarrollado (ecuaciones 1-10) requiere del conocimiento de las propiedades críticas $\left(T_{c}, P_{c}, Z_{c}\right)$ y el factor acéntrico de la sustancia pura. En la Tabla 4 se reporta los valores de las propiedades críticas y el factor acéntrico. Los valores reportados en esta Tabla para los LIs son obtenidos de Valderrama et al. (2015) y para alcoholes de la base de datos de la DIPPR (Daubert, 1998). La EdE es expresada en términos de solubilidad y como en toda ecuación cúbica entrega tres raíces (o soluciones) para una situación definida de presión y temperatura. Para ecuaciones cúbicas de estado los métodos más comúnmente usados son el numérico de Newton-Raphson y el algebraico de Cardano (1968). En este trabajo se usa el método de Cardano para la determinación de las raíces y como estamos en la zona de saturación se obtendrán tres raíces reales. La raíz mayor positiva corresponde a la fracción molar de líquido iónico en la fase 2 (a la derecha de la temperatura consoluta), mientras que la raíz menor positiva corresponde a la fracción molar de líquido iónico en la fase 1 (izquierda de la temperatura consoluta). En la temperatura consoluta, que corresponde al punto máximo en la Figura 2, resulta en una raíz positiva real y dos complejas conjugadas. 
Tabla 3: Rangos de temperatura y de concentración para cada una de las mezclas estudiadas, a 1 bar de presión

\begin{tabular}{|c|c|c|c|c|c|c|c|}
\hline No. & Mezclas de LI en alcohol & $\mathrm{N}$ & $\mathrm{T}_{\min }(\mathrm{K})$ & $\mathrm{T}_{\text {máx }}(\mathrm{K})$ & $x_{1, \text { mín }}$ & $\mathrm{X}_{1, \text { máx }}$ & Ref. \\
\hline 1 & [C6mim] [BF4]+1-butanol & 19 & 282.77 & 301.70 & 0.0085 & 0.4462 & 1 \\
\hline 2 & [C6mim] [BF4]+1-pentanol & 27 & 282.66 & 313.15 & 0.0068 & 0.5834 & 1 \\
\hline 3 & [C6mim] [BF4]+1-hexanol & 25 & 294.65 & 325.45 & 0.0080 & 0.6135 & 1 \\
\hline 4 & [C6mim] [BF4]+isobutanol & 18 & 284.50 & 304.40 & 0.0055 & 0.4452 & 1 \\
\hline 5 & {$[\mathrm{C} 8 \mathrm{mim}][\mathrm{BF} 4]+1-$ butanol } & 15 & 276.97 & 279.49 & 0.0158 & 0.1567 & 1 \\
\hline 6 & [C8mim] [BF4]+1-pentanol & 26 & 279.86 & 286.25 & 0.0113 & 0.2750 & 1 \\
\hline 7 & [C8mim] [BF4]+1-hexanol & 33 & 279.97 & 297.75 & 0.0029 & 0.3920 & 1 \\
\hline 8 & [C8mim] [BF4]+isobutanol & 20 & 277.37 & 284.47 & 0.0155 & 0.2475 & 1 \\
\hline 9 & {$[\mathrm{C} 8 \mathrm{mim}][\mathrm{BF} 4]+1$-octanol } & 17 & 301.75 & 312.67 & 0.0099 & 0.3710 & 1 \\
\hline 10 & [C4mim] [BF4]+1-propanol & 19 & 293.92 & 312.33 & 0.0095 & 0.4019 & 2 \\
\hline 11 & {$[\mathrm{C} 4 \mathrm{mim}][\mathrm{BF} 4]+1-$ butanol } & 25 & 298.54 & 329.92 & 0.0043 & 0.6444 & 2 \\
\hline 12 & [C4mim] [BF4]+1-pentanol & 19 & 303.92 & 346.03 & 0.0026 & 0.7037 & 2 \\
\hline 13 & [bmpy] [BF4]+1-propanol & 10 & 292.20 & 305.60 & 0.0146 & 0.3952 & 2 \\
\hline 14 & [bmpy][BF4]+1-butanol & 12 & 293.90 & 321.90 & 0.0099 & 0.5700 & 2 \\
\hline 15 & [C4mim] [PF6]+1-propanol & 20 & 297.32 & 359.27 & 0.0013 & 0.6840 & 2 \\
\hline 16 & [C4mim] [PF6]+1-butanol & 21 & 296.98 & 364.88 & 0.0105 & 0.7777 & 2 \\
\hline 17 & [C4mim] [PF6]+1-pentanol & 11 & 301.36 & 363.44 & 0.0020 & 0.8895 & 2 \\
\hline 18 & [C2mim] [PF6]+etanol & 17 & 321.27 & 351.43 & 0.0044 & 0.4947 & 3 \\
\hline 19 & [C2mim] [PF6]+1-propanol & 11 & 325.45 & 368.03 & 0.0048 & 0.7057 & 3 \\
\hline 20 & [C2mim] [PF6]+2-propanol & 13 & 324.30 & 354.41 & 0.0022 & 0.6800 & 3 \\
\hline 21 & [C2mim] [PF6]+1-butanol & 18 & 328.15 & 389.65 & 0.0026 & 0.7428 & 3 \\
\hline 22 & [C2mim] [PF6]+2-butanol & 7 & 346.03 & 371.04 & 0.4540 & 0.6789 & 3 \\
\hline 23 & [C2mim] [PF6]+alcohol terc-butílico & 6 & 326.15 & 354.35 & 0.5200 & 0.6852 & 3 \\
\hline 24 & [C2mim] [PF6]+3-metil-1-butanol & 8 & 332.18 & 403.20 & 0.6468 & 0.8050 & 3 \\
\hline 25 & [bmpy] [bti]+1-butanol & 8 & 291.70 & 302.80 & 0.0255 & 0.3601 & 3 \\
\hline 26 & [bmpy] [bti]+1-hexanol & 12 & 299.60 & 335.00 & 0.0103 & 0.6174 & 4 \\
\hline 27 & [hpy] [bti]+1-hexanol & 8 & 297.70 & 309.70 & 0.0200 & 0.3337 & 4 \\
\hline 28 & [hmmpy] [bti]+1-hexanol & 10 & 295.30 & 312.00 & 0.0147 & 0.4100 & 4 \\
\hline
\end{tabular}

Tabla 4: Propiedades críticas del componente puro para todas las sustancias involucradas en este estudio

\begin{tabular}{|l|c|c|c|c|}
\hline Líquido iónico & $\mathrm{T}_{\mathrm{c}}(\mathrm{K})$ & $\mathrm{P}_{\mathrm{c}}(\mathrm{bar})$ & $\mathrm{Z}_{\mathrm{c}}$ & $\omega$ \\
\hline [C6mim] [BF4] & 689.98 & 17.94 & 0.2406 & 0.9625 \\
\hline$[\mathrm{C} 8 \mathrm{mim}][\mathrm{BF} 4]$ & 736.99 & 16.02 & 0.2310 & 1.0287 \\
\hline$[\mathrm{C} 4 \mathrm{mim}][\mathrm{PF}]$ & 719.39 & 17.28 & 0.2203 & 0.7917 \\
\hline [C4mim] [BF4] & 643.18 & 20.38 & 0.2496 & 0.8877 \\
\hline$[\mathrm{C} 2 \mathrm{mim}][\mathrm{PF}]$ & 674.01 & 19.52 & 0.2258 & 0.7083 \\
\hline$[\mathrm{bmpy}][\mathrm{BF} 4]$ & 625.79 & 18.90 & 0.2556 & 0.8924 \\
\hline$[$ bmpy] [bti] & 1240.48 & 25.53 & 0.2571 & 0.3160 \\
\hline [hpy] [bti] & 1252.31 & 23.93 & 0.2522 & 0.3383 \\
\hline [hmmpy] [bti] & 1279.15 & 20.78 & 0.2362 & 0.4747 \\
\hline 1-butanol & 563.00 & 44.14 & 0.2580 & 0.5895 \\
\hline 1-pentanol & 588.10 & 38.97 & 0.2600 & 0.5731 \\
\hline 1-hexanol & 610.30 & 34.17 & 0.261 & 0.5764 \\
\hline isobutanol & 547.80 & 42.95 & 0.258 & 0.5857 \\
\hline 1-octanol & 652.50 & 27.77 & 0.254 & 0.5829 \\
\hline 1-propanol & 536.80 & 51.69 & 0.252 & 0.6204 \\
\hline etanol & 514.00 & 61.37 & 0.241 & 0.6436 \\
\hline 2-propanol & 508.30 & 47.64 & 0.25 & 0.6669 \\
\hline 2-butanol & 536.20 & 42.02 & 0.254 & 0.5768 \\
\hline alcohol terc-butílico & 506.20 & 39.72 & 0.26 & 0.6152 \\
\hline 3-metil-1-butanol & 577.20 & 39.30 & 0.268 & 0.5890 \\
\hline
\end{tabular}

En total se usan 455 datos experimentales que representan 28 mezclas binarias de líquido iónico en alcohol. El $71 \%$ de los datos (322 datos) son para la correlación mientras que el $29 \%$ de los datos (133 datos) para el proceso de predicción. Los datos seleccionados para el proceso de correlación y predicción son tomados de manera aleatoria (usando la función aleatoria de MS Excel). El proceso de correlación se realiza con el modelo de solubilidad (ecuaciones 1-10) y con $\mathrm{k}_{12}=0$ (sin ningún parámetro de interacción). El proceso de ajuste se realiza mediante regresión no lineal y el algoritmo de Lasdon et al. (1978) incorporado en Solver de MS Excel y con la siguiente función objetivo a minimizar: 


$$
\left|\% \Delta X_{1}\right|=\frac{100}{N} \cdot \frac{\left|X_{1, i}{ }^{\text {cal }}-X_{1, i}^{\text {exp }}\right|}{X_{1, i}{ }^{\text {exp }}}
$$

La función objetivo descrita en la ecuación 11 representa la desviación absoluta de la fracción molar de líquido iónico $\left(x_{1}\right)$ en la fase 1 . Además, esta función objetivo ha sido usada con éxito en diferentes aplicaciones en la determinación de parámetros de modelos termodinámicos (Khosharay et al., 2018; Cardona et al., 2019). En esta ecuación los superíndices "exp" y "cal" indican la fracción molar de líquido iónico experimental y calculada con el modelo de solubilidad y $\mathrm{N}$ es el número de datos. Luego de obtener los parámetros óptimos es necesario realizar un análisis estadístico que permita realizar inferencias (bondad y robustez de un modelo) para cada mezcla estudiada (Valderrama y Alvarez, 2005). Lo anterior implica, la determinación de la desviación absoluta máxima y la desviación relativa dada por las siguientes ecuaciones:

$$
\begin{aligned}
& \% \Delta x_{1}=\frac{100}{N} \cdot \sum_{i=1}^{N}\left(\frac{x_{1, i}{ }^{\text {cal }}-X_{1, i}{ }^{\text {exp }}}{x_{1, i}{ }^{\text {exp }}}\right) \\
& \left|\% \Delta \mathrm{x}_{1}\right|_{\max }=\text { máx.valor de } 100 \cdot \frac{\left|\mathrm{x}_{1, \mathrm{i}}{ }^{\text {cal }}-\mathrm{x}_{1, \mathrm{i}}{ }^{\text {exp }}\right|}{\mathrm{x}_{1, \mathrm{i}}{ }^{\text {exp }}}
\end{aligned}
$$

Luego, con los valores determinados de los parámetros se analiza la capacidad predictiva del modelo para determinar la solubilidad en aquellos casos en que no se usaron los datos experimentales en la obtención de los parámetros. Teniendo los parámetros ajustados se determina el coeficiente de interacción $\mathrm{k}_{12}\left(\mathrm{k}_{12} \neq 0\right)$ para cada mezcla. Este proceso se realiza utilizando la ecuación 11 como función objetivo a minimizar y observando las desviaciones medias y máximas como parámetros estadísticos para determinar la bondad del modelo (Valderrama y Alvarez, 2005).

\section{RESULTADOS Y DISCUSIÓN}

Las mezclas binarias reportadas en la Tabla 3 han sido agrupadas de acuerdo con el tipo de líquido iónico. Posteriormente, se estiman los 9 parámetros del modelo para cada mezcla con $\mathrm{R}^{*}$ constante. La Tabla 5 se muestra los parámetros del modelo con $\mathrm{k}_{12}=0$.

Tabla 5: Parámetros óptimos obtenidos durante el proceso de correlación $\left(R^{\star}=1.8321 \mathrm{E}-06\right.$ bar/K)

\begin{tabular}{|l|l|c|c|c|c|c|c|c|c|}
\hline $\mathrm{LI}$ & $\phi_{0}$ & $\phi_{1}$ & $\beta_{0}$ & $\beta_{1}$ & $\gamma_{0}$ & $\gamma_{1}$ & $\mathrm{~h}_{1}$ & $\mathrm{~h}_{2}$ & $\mathrm{~h}_{3}$ \\
\hline [C6mim][BF4] & -51303.23 & -35315.69 & 79837.60 & -301253.74 & -129.91 & -4.88 & -200.68 & 2583.07 & -8400.40 \\
\hline [C8mim][BF4] & -14059.41 & -31324.24 & 16795.51 & -59069.57 & -116.59 & -4.88 & -199.25 & 2599.09 & -8536.65 \\
\hline [C4mim][BF4] & -208849.36 & -36629.38 & 13748.86 & -42699.24 & -106.10 & -4.87 & -158.18 & 2036.09 & -6673.46 \\
\hline [C4mpy][BF4] & -113480.02 & -36473.71 & 34929.56 & -123994.57 & -127.84 & -4.88 & -207.05 & 2607.62 & -8295.45 \\
\hline [C4mim][PF6] & -156198.08 & -37112.05 & 67521.99 & -249834.32 & -210.96 & -4.93 & -206.17 & 2599.06 & -8280.18 \\
\hline [C2mim][PF6] & -438955.50 & -36754.08 & 26553.85 & -59987.05 & -125.80 & -4.88 & -262.66 & 3264.35 & -10247.99 \\
\hline [bmpy][bti] & -3773.09 & -28572.49 & 59062.61 & -215794.05 & -121.89 & -4.88 & -180.68 & 2047.28 & -5564.82 \\
\hline [hpy][bti] & -9434.09 & -35395.76 & 228602.00 & -873493.12 & -102.64 & -4.87 & -204.14 & 2595.86 & -8336.58 \\
\hline [hmpy][bti] & -75270.31 & -36904.40 & 92782.97 & -355466.24 & -98.64 & -4.86 & -195.02 & 2584.83 & -8628.08 \\
\hline
\end{tabular}

En la Tabla 5 los parámetros $\phi_{1}, \gamma_{1}, h_{1}, h_{2}$ y $h_{3}$ fueron considerados constantes (y luego recalculados como constantes) ya que su variación no genera cambios significativos en la función objetivo; ni en el promedio (ecn. 12), ni en el máximo (ecn. 13). Esta función objetivo (12) varía de $6.8 \%$ (valores óptimos) a $9.1 \%$ ( $\phi_{1}, \gamma_{1}$, $h_{1}, h_{2}$ y $h_{3}$ constantes). Con el propósito de tener estos parámetros constantes, se realiza de nuevo el proceso de optimización usando la función objetivo dado por la ecuación 11 y se recalcula los valores de los parámetros $\phi_{0}, \beta_{0}, \beta_{1}$ y $\gamma_{0}$. En resumen, los valores de los parámetros $\phi_{0}, \beta_{0}, \beta_{1}$ y $\gamma_{0}$ son específicos de cada mezcla mientras que $\phi_{1}, \gamma_{1}, h_{1}, h_{2} y_{h}$ son valores constantes que no dependen de la naturaleza de la mezcla. En la Tabla 6, se muestra los resultados de los parámetros óptimos $\phi_{0}, \beta_{0}, \beta_{1}$ y $\gamma_{0}$ teniendo como constante $\phi_{1}, \gamma_{1}, h_{1}, h_{2}$ y $h_{3}$. Es importante aclarar que el valor de $R^{*}$ sigue siendo el mismo valor reportado en la Tabla 5: $R^{*}=1.8321 \mathrm{E}-06 \mathrm{bar} / \mathrm{K}$. Con los valores estimados de los parámetros se determina para cada mezcla el parámetro de interacción $k_{12}$ (ver ecuación 2) y los resultados se muestran en la Tabla 7. En esta Tabla se reporta las desviaciones absolutas, relativas y máximas (ver ecuaciones 11-13) para cada una de las mezclas. 
Tabla 6: Parámetros óptimos $\phi_{0}, \beta_{0}, \beta_{1}$ y $\gamma_{0}$ teniendo como constante $\phi_{1}, \gamma_{1}, h_{1}, h_{2}$ y $h_{3}$ para todas las mezclas analizadas $\left(\mathrm{R}^{*}=1.8321 \mathrm{E}-06 \mathrm{bar} / \mathrm{K}\right)$.

\begin{tabular}{|c|c|c|c|c|c|c|c|c|c|}
\hline $\mathrm{LI}$ & $\phi_{0}$ & $\phi_{1}$ & $\beta_{0}$ & $\beta_{1}$ & $\gamma_{0}$ & $\gamma_{1}$ & $h_{1}$ & $\mathrm{~h}_{2}$ & $h_{3}$ \\
\hline$[\mathrm{C} 6 \mathrm{mim}][\mathrm{BF} 4]$ & -61536.88 & \multirow{9}{*}{-39461.27} & 80237.448 & -302497.49 & -145.68 & \multirow{9}{*}{-4.88} & \multirow{9}{*}{-197.23} & \multirow{9}{*}{2506.78} & \multirow{9}{*}{-8047.36} \\
\hline$[\mathrm{C} 8 \mathrm{mim}][\mathrm{BF} 4]$ & -17888.71 & & 18157.34 & -66584.09 & -76.40 & & & & \\
\hline [C4mim][BF4] & -171736.91 & & 15171.45 & -40829.68 & -126.47 & & & & \\
\hline [C4mpy][BF4] & -113211.57 & & 36478.63 & -128832.73 & -129.73 & & & & \\
\hline [C4mim][PF6] & -168777.99 & & 60051.39 & -216848.79 & -265.88 & & & & \\
\hline [C2mim][PF6] & -274275.68 & & 25899.09 & -57349.88 & -114.84 & & & & \\
\hline [bmpy][bti] & -3683.43 & & 58629.45 & -217129.33 & -113.03 & & & & \\
\hline [hpy][bti] & -9477.56 & & 228857.28 & -874708.68 & -101.49 & & & & \\
\hline [hmpy][bti] & -58939.71 & & 92101.68 & -352771.98 & -98.57 & & & & \\
\hline
\end{tabular}

Tabla 7: Desviaciones relativas y absolutas con $\mathrm{k}_{12}=0$ y $\mathrm{k}_{12} \neq 0$ a partir de los valores de los parámetros óptimos estimados de la Tabla 6.

\begin{tabular}{|c|c|c|c|c|c|c|c|c|}
\hline \multirow{2}{*}{ No. } & \multirow{2}{*}{ mezcla binaria: LI+alcohol } & \multicolumn{3}{|c|}{$\mathrm{k}_{12}=0$} & \multicolumn{4}{|c|}{$\mathrm{k}_{12} \neq 0$} \\
\hline & & $\% \Delta \mathrm{x}_{1}$ & $\left|\% \Delta x_{1}\right|$ & $\left|\% \Delta \mathrm{x}_{1}\right|_{\max }$ & $\mathrm{k}_{\mathrm{ij}}$ & $\% \Delta \mathrm{x}_{1}$ & $\% \Delta \mathrm{x}_{1}$ & $\left|\% \Delta \mathrm{x}_{1}\right| \max$ \\
\hline 1 & [C6mim] [BF4]+1-butanol & 0.21 & 2.43 & 6.28 & -0.0614 & 0.08 & 2.42 & 6.27 \\
\hline 2 & [C6mim] [BF4]+1-pentanol & -3.04 & 4.87 & 9.50 & -0.0072 & -0.32 & 3.00 & 6.62 \\
\hline 3 & [C6mim] [BF4]+1-hexanol & -2.44 & 6.90 & 25.26 & 0.2961 & -2.28 & 6.90 & 26.05 \\
\hline 4 & [C6mim] [BF4]+isobutanol & 1.17 & 2.61 & 11.43 & 0.0146 & 1.03 & 2.59 & 11.04 \\
\hline 5 & [C8mim] [BF4]+1-butanol & 2.03 & 4.52 & 16.87 & 0.0296 & 0.35 & 3.96 & 17.28 \\
\hline 6 & [C8mim] [BF4]+1-pentanol & -5.04 & 7.10 & 38.52 & -0.1003 & -3.40 & 6.64 & 38.45 \\
\hline 7 & [C8mim] [BF4]+1-hexanol & -4.83 & 4.07 & 33.82 & -0.0481 & -4.44 & 3.87 & 33.81 \\
\hline 8 & [C8mim] [BF4]+isobutanol & -2.20 & 3.76 & 17.78 & 0.0480 & -3.21 & 3.28 & 18.20 \\
\hline 9 & [C8mim] [BF4]+1-octanol & -20.07 & 23.48 & 64.20 & -0.9330 & -14.11 & 15.69 & 39.45 \\
\hline 10 & [C4mim] [BF4]+1-propanol & 3.73 & 5.25 & 32.75 & -0.0157 & 3.42 & 5.17 & 31.86 \\
\hline 11 & [C4mim] [BF4]+1-butanol & 3.87 & 13.74 & 62.44 & 0.0310 & 4.24 & 13.67 & 63.30 \\
\hline 12 & [C4mim] [BF4]+1-pentanol & -12.87 & 13.23 & 28.42 & -0.0568 & -3.09 & 11.00 & 26.70 \\
\hline 13 & [bmpy] [BF4]+1-propanol & 2.00 & 3.46 & 8.72 & 0.0630 & 1.90 & 3.44 & 8.70 \\
\hline 14 & [bmpy][BF4]+1-butanol & -4.07 & 4.19 & 9.81 & -0.1225 & -1.95 & 4.18 & 7.41 \\
\hline 15 & [C4mim] [PF6]+1-propanol & -6.84 & 23.98 & 95.78 & -0.8654 & -5.92 & 23.49 & 94.31 \\
\hline 16 & [C4mim] [PF6]+1-butanol & -5.11 & 9.36 & 53.05 & -0.9401 & -5.58 & 9.82 & 56.08 \\
\hline 17 & [C4mim] [PF6]+1-pentanol & -2.49 & 2.51 & 4.98 & -0.8625 & -2.49 & 2.51 & 4.98 \\
\hline 18 & [C2mim] [PF6]+etanol & -1.82 & 35.08 & 82.03 & -0.9804 & -1.22 & 34.70 & 80.14 \\
\hline 19 & [C2mim] [PF6]+1-propanol & 0.92 & 13.13 & 35.55 & -0.9650 & 1.53 & 13.10 & 34.57 \\
\hline 20 & [C2mim] [PF6]+2-propanol & -11.05 & 13.83 & 40.11 & -0.9844 & -10.32 & 13.10 & 38.30 \\
\hline 21 & [C2mim] [PF6]+1-butanol & 2.94 & 20.80 & 49.59 & -0.7090 & 2.71 & 20.40 & 47.27 \\
\hline 22 & [C2mim] [PF6]+2-butanol & 0.18 & 3.88 & 7.86 & 0.0049 & 0.18 & 3.88 & 7.86 \\
\hline 23 & [C2mim] [PF6]+alcohol terc-butílico & 2.74 & 2.99 & 5.73 & 0.0291 & 2.74 & 2.99 & 5.73 \\
\hline 24 & [C2mim] [PF6]+3-metil-1-butanol & -6.95 & 6.95 & 11.14 & -0.0195 & -6.95 & 6.95 & 11.14 \\
\hline 25 & [bmpy] [bti]+1-butanol & -7.74 & 11.80 & 37.26 & 0.0007 & -2.07 & 8.67 & 20.61 \\
\hline 26 & [bmpy] [bti]+1-hexanol & -5.36 & 8.60 & 32.27 & -0.5329 & -3.03 & 7.17 & 32.09 \\
\hline 27 & [hpy] [bti]+1-hexanol & 0.40 & 0.61 & 2.30 & -0.1203 & 0.45 & 0.60 & 2.35 \\
\hline \multirow[t]{2}{*}{28} & [hmmpy] [bti]+1-hexanol & -2.74 & 1.62 & 6.12 & -0.0093 & -1.86 & 1.52 & 3.90 \\
\hline & Promedio Global & -3.02 & 9.10 & ---- & ---- & -1.91 & 8.38 & $\begin{array}{l}--- \\
\end{array}$ \\
\hline
\end{tabular}

En la Tabla 8, se muestra un resumen estadístico de las capacidades de correlación y predicción del modelo de solubilidad teniendo como constante los parámetros $\phi_{1}, \gamma_{1}, h_{1}, h_{2}$ y $h_{3}$ para todas las mezclas. En la Figura 3 se muestran los valores estimados y experimentales de la fracción molar de líquido iónico, demostrando una adecuada distribución entre las composiciones calculadas y los datos experimentales con un coeficiente de correlación mayor a 0.995. El modelo pese a presentar algunos valores con alta desviación genera resultados promedio aceptables. La Figura 4 muestra los valores estimados y experimentales de las solubilidades para diferentes mezclas binarias compuestas por un $\mathrm{LI}+$ alcohol a diferentes temperaturas. 
Tabla 8: Resumen de desviaciones usando el modelo de solubilidad con los valores $\phi_{1}, \gamma_{1}, h_{1}, h_{2}$ y $h_{3}$ constantes.

\begin{tabular}{|l|c|c|c|c|c|c|c|}
\hline \multicolumn{2}{|c|}{} & \multicolumn{2}{|c|}{ Desviación absoluta } & \multicolumn{2}{c|}{ Desviación relativa } & \multicolumn{2}{c|}{ Rangos de desviación } \\
\hline Proceso & $\mathrm{N}$ & promedio $\left|\% \Delta \mathrm{x}_{1}\right|$ & $\left|\% \Delta \mathrm{x}_{1}\right|_{\max }$ & promedio $\% \Delta \mathrm{x}_{1}$ & $\% \Delta \mathrm{x}_{1, \text { máx }}$ & $\begin{array}{c}\text { No. Datos con } \\
\left|\% \Delta \mathrm{x}_{1}\right|>10\end{array}$ & $\begin{array}{c}\text { No. Datos con } \\
\left|\% \Delta \mathrm{x}_{1}\right|>30\end{array}$ \\
\hline Correlación con $\mathrm{k}_{12}=0$ & 322 & 9.1 & 95.3 & -3.0 & 62.4 & $69(21.4 \%)$ & $27(8.4 \%)$ \\
\hline Predicción con $\mathrm{k}_{12}=0$ & 133 & 11.8 & 94.3 & -5.0 & 94.5 & $64(23.3 \%)$ & $17(12.8 \%)$ \\
\hline Correlación con $\mathrm{k}_{12} \neq 0$ & 322 & 8.4 & 94.3 & -1.9 & 63.3 & $64(19.9 \%)$ & $24(7.5 \%)$ \\
\hline Predicción con $\mathrm{k}_{12} \neq 0$ & 133 & 10.5 & 92.5 & -3.3 & 92.5 & $29(21.8 \%)$ & $15(11.3 \%)$ \\
\hline
\end{tabular}
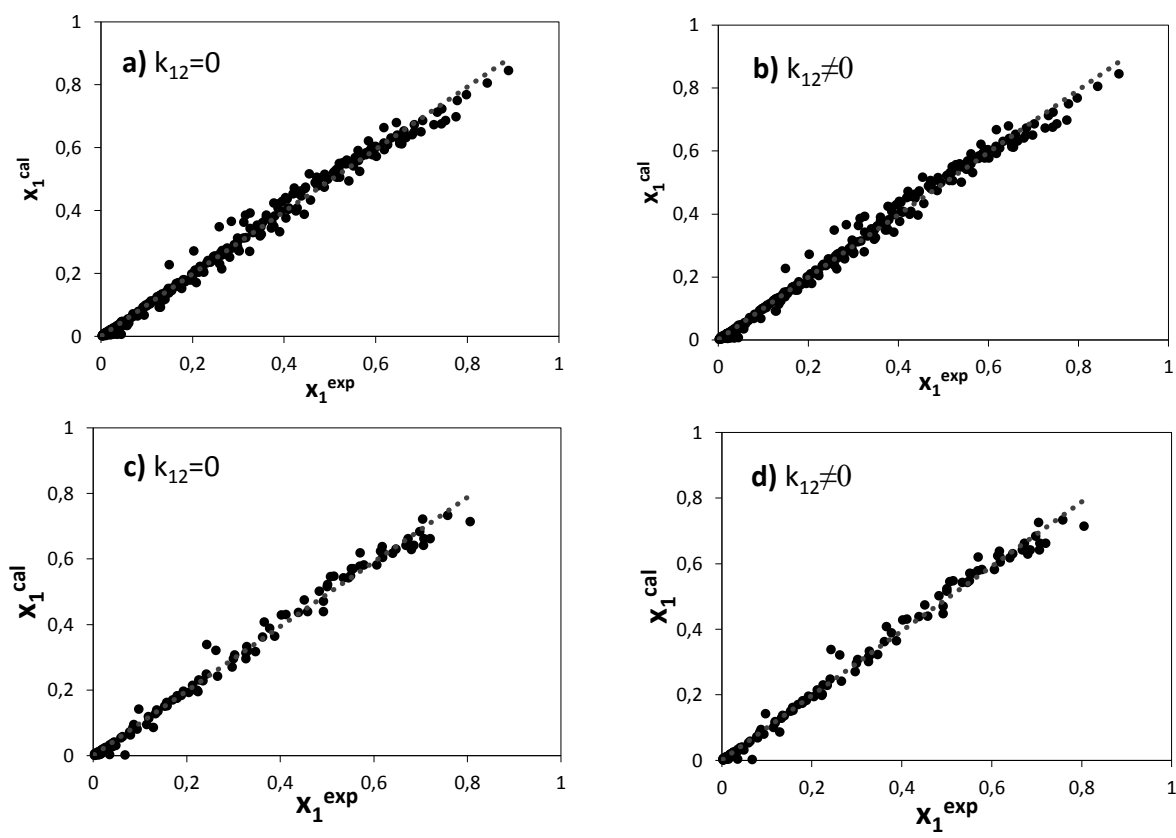

Fig. 3: Concentración de líquido iónico calculados vs experimentales usando el modelo de ecuación de estado de solubilidad (ecuaciones 1-10). a) y b) corresponden al proceso de correlación y c) y d) al proceso de predicción.
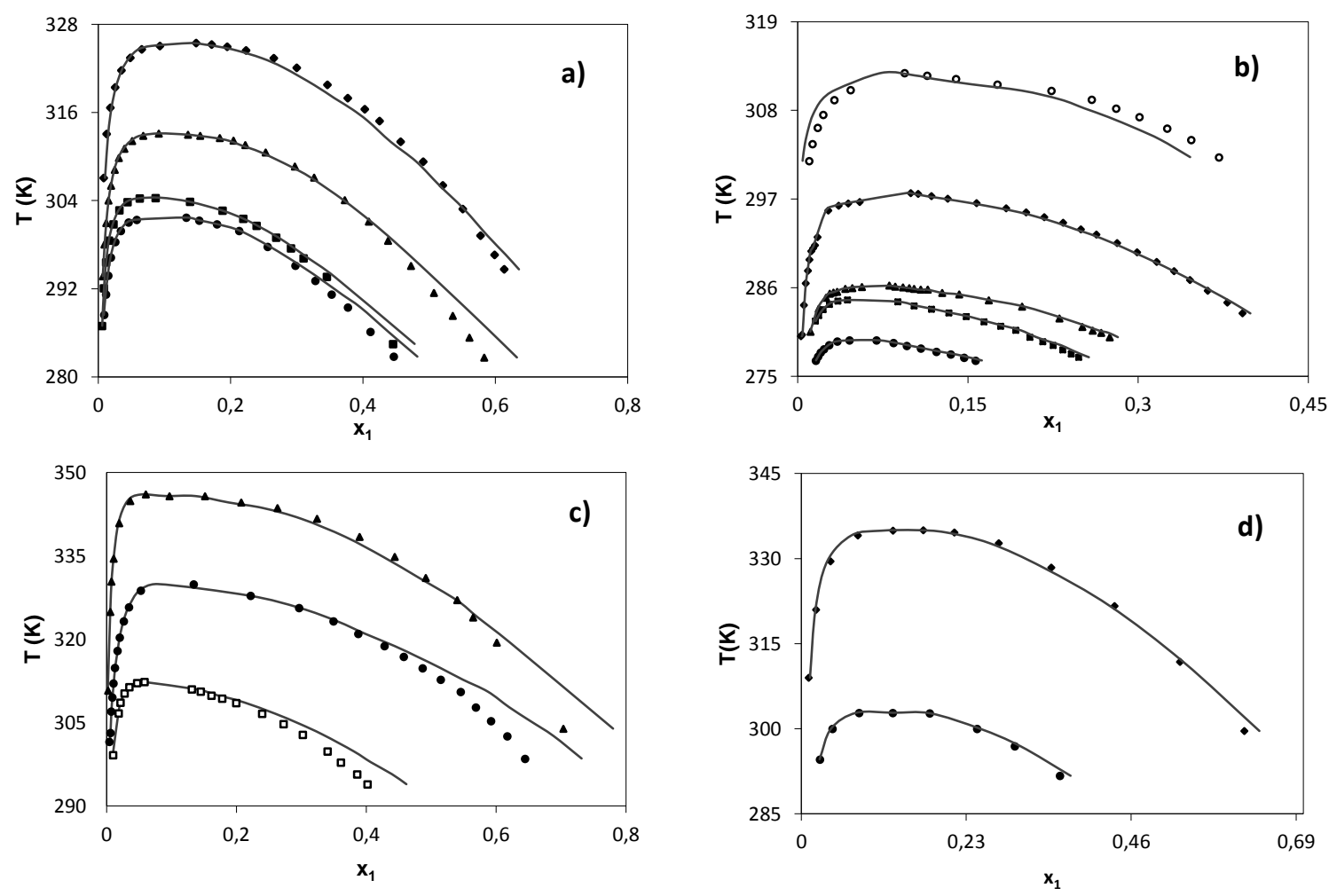

Fig. 4: Comparación de las solubilidades calculadas y experimentales. a) [C6mim][BF4]+alcohol; b) [C8mim][BF4]+alcohol; c) [C4mim][BF4]+alcohol; d) [bmpy] [bti]+alcohol: •: 1-butanol, a: isobutanol; $\boldsymbol{\Delta}$ : 1-pentanol; : 1-hexanol; 口: 1-propanol; ○: 1-octanol. La línea sólida es la estimación del modelo de solubilidad (ecuaciones 1-10). 
Altas desviaciones se presentan en las mezclas binarias de [C4mim][PF6] y [C2mim][PF6] (ver Tabla 7) que corresponden a concentraciones bajas (fase 1, localizado a la izquierda del diagrama PxT de la Figura 2). De acuerdo con el trabajo experimental realizado sobre estas mezclas (Huo et al.,2008; Domańska y Marciniak, 2004), altos contenidos de agua en su preparación generan que la temperatura consoluta superior (Tu) disminuya hasta 10K (Marsh et al., 2004). Lo anterior origina variaciones considerables en la curva PxT, generando altas desviaciones en la correlación (Domańska y Marciniak, 2004). Algunos errores erráticamente altos para la desviación máxima pueden ser observados en la Tabla 7 y resumidos en la Tabla 8 . Aunque puede haber errores experimentales que justifiquen estas altas desviaciones, el modelo necesita ser explorado en más profundidad.

\section{DISCUSIÓN FINAL}

De acuerdo con la revisión de la literatura realizada en este trabajo, la aplicación del concepto de similitud geométrica usando ecuaciones de estado no ha sido aplicado para modelar la solubilidad mutua en mezcla parcialmente miscibles. Por lo tanto, el presente artículo muestra una novedad y una alternativa para modelarlo, y que debe ser mejor explorada en diferentes aplicaciones. Otros modelos de solubilidad en mezclas parcialmente miscibles de líquidos iónicos han sido propuestas en la literatura y se resumen en la Tabla 9, incluyendo los modelos NRTL y Weimar y Prausnitz. Además, en esta Tabla (columna 3) $n_{p}$ corresponde al número de parámetros del modelo, $\mathrm{n}_{\mathrm{m}}$ (cuarta columna) es el número total de mezclas usadas por los autores en el modelado y $n_{d}$ es el número de datos experimentales usados en el proceso de ajuste. En la sexta columna de la Tabla 9 hace referencia a la mezcla que forma el líquido iónico con el alcohol. Los alcoholes son los mismos reportados en la Tabla 3. Los modelos comparados en la Tabla 9 no realizan predicciones satisfactorias en condiciones diferentes al ajuste y de acuerdo con Marsh et al. (2004) el aporte en el desarrollo de modelos de solubilidad se debe centrar en su capacidad predictiva. En este trabajo se obtienen resultados similares tanto en correlación como predicción demostrando la robustez del modelo. Es importante aclarar que el modelo desarrollado en esta investigación presenta 4 parámetros ajustables y 6 constantes para todas las mezclas estudiadas con desviaciones absolutas similares tanto en el proceso de correlación como predicción.

Tabla 9: Desviaciones $|\% \Delta x|$ y máx. $|\% \Delta x|$ para la solubilidad en líquidos iónicos. (1) Bai et al. (2011), (2) Huo et al. (2008), (3) Domańska y Marciniak (2004) y (4) Crosthwaite et al. (2006).

\begin{tabular}{|c|c|c|c|c|c|c|c|c|c|c|}
\hline \multirow[b]{2}{*}{ № } & \multirow{2}{*}{ Modelo } & \multirow{2}{*}{$\mathrm{n}_{\mathrm{p}}$} & \multirow[b]{2}{*}{$\mathrm{n}_{\mathrm{m}}$} & \multirow{2}{*}{$\mathrm{n}_{\mathrm{d}}$} & \multirow[b]{2}{*}{ LI } & \multirow[b]{2}{*}{$\Delta \mathrm{T}(\mathrm{K})$} & \multicolumn{3}{|c|}{ 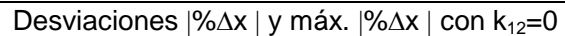 } & \multirow{2}{*}{ Ref. } \\
\hline & & & & & & & Literatura & Correlación & Predicción & \\
\hline 1 & NRTL & 10 & 9 & 200 & $\begin{array}{l}{[\mathrm{C} 8 \mathrm{mim}][\mathrm{BF} 4]} \\
{[\mathrm{C} 6 \mathrm{mim}][\mathrm{BF} 4]}\end{array}$ & $276-325$ & $\begin{array}{c}1.4 \% \\
\text { (máx. 18\%) }\end{array}$ & $\begin{array}{c}6.6 \% \text { (máx. } \\
64.2 \% \text { ) }\end{array}$ & $\begin{array}{c}5.96 \\
\text { (máx. 56\%) } \\
\end{array}$ & 1 \\
\hline 2 & NRTL & 6 & 6 & 116 & $\begin{array}{l}{[\mathrm{C} 4 \mathrm{mim}][\mathrm{PF} 6]} \\
{[\mathrm{C} 4 \mathrm{mim}][\mathrm{BF} 4]}\end{array}$ & 293-365 & $\begin{array}{c}2.5 \% \\
\text { (máx. } 70 \% \text { ) }\end{array}$ & $\begin{array}{c}7.9 \% \\
\text { (máx. 95\%) }\end{array}$ & $\begin{array}{c}13.4 \% \\
\text { (máx. } 94.7 \% \text { ) }\end{array}$ & 2 \\
\hline 3 & $\begin{array}{l}\text { Weimar y } \\
\text { Prausnitz }\end{array}$ & 10 & 7 & 80 & [C2mim][PF6] & $321-403$ & $\begin{array}{c}3.0 \% \\
\text { (máx. } 80 \% \text { ) }\end{array}$ & $\begin{array}{c}13.8 \% \text { (máx. } \\
82.0 \% \text { ) }\end{array}$ & $\begin{array}{c}12.1 \% \\
\text { (máx. } 88.0 \% \text { ) }\end{array}$ & 3 \\
\hline 4 & NRTL & 4 & 9 & 90 & $\begin{array}{l}\text { [bmpy] [bti] } \\
\text { [hpy] [bti] } \\
\text { [hmmpy] [bti] }\end{array}$ & 291-335 & $\begin{array}{l}10.3 \% \\
\text { (máx. } \\
89 \% \text { ) }\end{array}$ & $\begin{array}{c}5.7 \% \text { (máx. } \\
32.3 \% \text { ) }\end{array}$ & $\begin{array}{c}4.48 \% \\
\text { (máx. 17\%) }\end{array}$ & 4 \\
\hline
\end{tabular}

\section{CONCLUSIONES}

De acuerdo con los resultados presentados en este artículo, se pueden extraer las siguientes cuatro conclusiones: 1) El concepto de similitud geométrica representa una alternativa para modelar diferentes propiedades físicas, tal es el caso de la solubilidad de mezclas parcialmente miscibles de líquidos iónicos en alcoholes; 2) El modelo de solubilidad del tipo ecuación de estado genera resultados aceptables tanto en el proceso de correlación y predicción de mezclas binarias de líquidos iónicos en alcoholes; 3) La comparación del modelo desarrollado en esta investigación frente a otros en la literatura tiene la ventaja de ser simple y generar resultados predictivos aceptables; 4) El modelo empírico desarrollado en esta investigación podrá ser utilizado en aplicaciones industriales en el diseño y optimización de procesos de extracción líquido-líquido y educación en ingeniería y ciencias básicas.

\section{AGRADECIMIENTOS}

Los autores agradecen a la Comisión Nacional de Investigación Científica y Tecnológica (CONICYT) por su apoyo a través del Proyecto Anillo ACM 170005 y agradecen el soporte computacional del Centro de Información Tecnológica de La Serena (Chile). LFC agradece a la Universidad Pontificia Bolivariana y al programa de Colciencias "Doctorados Nacionales-convocatoria 727 del 2015" por su apoyo durante la elaboración de este trabajo. CAF agradece al Departamento de Física de la Universidad de Concepción (Chile) por su apoyo durante la elaboración de este trabajo. 


\section{NOTACIÓN}

\section{Símbolos}

\begin{tabular}{|c|c|}
\hline$a, b, c$ & Parámetros de la ecuación de estado definidos en la Tablas 1 y 2 \\
\hline$h_{1}, h_{2}, h_{3}$ & Parámetros de la ecuación de Soave definidos en la ecuación 10 \\
\hline $\mathrm{n}_{\mathrm{p}}$ & Número de parámetros ajustables del modelo (ver Tabla 9) \\
\hline $\mathrm{n}_{\mathrm{m}}$ & Número de mezclas evaluadas por los autores (ver Tabla 9) \\
\hline$n_{d}$ & Número total de datos experimentales usados en el ajuste (ver Tabla 9) \\
\hline $\mathrm{N}$ & Número de datos \\
\hline $\mathrm{P}$ & Presión (bar) \\
\hline $\mathrm{R}^{*}$ & Constante para todas las mezclas definido en las ecuaciones 6-8 (bar/K) \\
\hline $\mathrm{T}$ & Temperatura $(\mathrm{K})$ \\
\hline $\mathrm{v}$ & Volumen molar $\left(\mathrm{mol} / \mathrm{cm}^{3}\right)$ \\
\hline$x$ & Fracción molar de líquido \\
\hline Z & Factor de compresibilidad \\
\hline \multicolumn{2}{|c|}{ Abreviaciones } \\
\hline $\mathrm{EdE}$ & Ecuación de estado \\
\hline ELL & Extracción Líquido-Líquido \\
\hline $\mathrm{LI}$ & Líquido iónico \\
\hline MS & Microsoft \\
\hline PTV & Patel-Teja-Valderrama \\
\hline vdW & van der Waals \\
\hline \multicolumn{2}{|c|}{ Letras Griegas } \\
\hline$\alpha$ & Función alfa (ver Tabla 2 y ecuación 9) \\
\hline$\% \Delta$ & Porcentaje de desviación \\
\hline$\rho$ & Densidad másica $\left(\mathrm{g} / \mathrm{cm}^{3}\right)$ \\
\hline$\phi, \beta, \gamma$ & Parámetros del modelo de solubilidad definidas en las ecuaciones 6-8 \\
\hline$\omega$ & Factor acéntrico \\
\hline \multicolumn{2}{|c|}{ Super/Subíndices } \\
\hline cal & Calculado \\
\hline c & Crítica \\
\hline $\exp$ & Experimental \\
\hline $\mathrm{i}, \mathrm{j}$ & Componentes i y j \\
\hline L & Fase líquida \\
\hline máx. & Máximo valor \\
\hline mín. & Mínimo valor \\
\hline
\end{tabular}

\section{REFERENCIAS}

Bai J.; P. Pan; X. Shuqian y M. Peisheng, Measurement and study on the liquid-liquid equilibrium of ionic liquids with aliphatic alcohols, Journal of Chemical \& Engineering Data, 56(11), 3963-3970 (2011).

Cardano, G., Ars magna or the rules of algebra, New York: Dover Publications (1968).

Cardona, L.F.; L.A. Forero y J.A. Velásquez, Modelamiento de la Viscosidad con Base en una Ecuación Cúbica $\mu$ TP del Tipo Peng-Robinson, Inf. Tecnol., 30(4), en prensa (2019).

Crosthwaite, J.M.; M.J. Muldoon; S.N. Aki; E.J. Maginn y J.F. Brennecke, Liquid phase behavior of ionic liquids with alcohols: experimental studies and modeling, The Journal of Physical Chemistry B., 110(18), 9354-9361 (2006).

Danesh, A.; Xu, D.; Todd, A. C. Comparative Study of Cubic Equations of State for Predicting Phase Behavior and Volumetric Properties of Injection Gas-Reservoir Oil Systems, Fluid Phase Equilib., 63, 259-278 (1991)

Danesh, A., PVT and Phase Behaviour of Petroleum Reservoir Fluids, Amsterdam: Elsevier (1998).

Daubert, T.E., Evaluated equation forms for correlating thermodynamic and transport properties with temperature, Industrial \& Engineering Chemistry Research, 37(8), 3260-3267 (1998). 
Domańska, U. y A. Marciniak, Solubility of ionic liquid [emim][PF6] in alcohols, The Journal of Physical Chemistry B., 108(7), 2376-2382 (2004).

Fan, T.B. y L.S. Wang, A viscosity model based on Peng-Robinson equation of state for light hydrocarbon liquids and gases, Fluid Phase Equil., 247(1-2), 59-69 (2006).

Guo, X.Q.; C.Y. Sun; S.X. Rong; G.J. Chen y T.M. Guo, Equation of state analog correlations for the viscosity and thermal conductivity of hydrocarbons and reservoir fluids, Journal of Petroleum Science and Engineering, 6 (1), 15-27 (2001).

Henley, E.J. y J.D. Seader, Operaciones de separación por etapas de equilibrio en ingeniería química, México: Reverté (2000).

Hekayati, J.; A. Roosta y J. Javanmardi. Thermodynamic Modeling of Refrigerant Solubility in lonic Liquids Using Original and e-Modified Sanchez-Lacombe Equation, Fluid Phase Equilib., 403, 14-22 (2015).

Huo, Y.; S. Xia y P. Ma, Solubility of alcohols and aromatic compounds in imidazolium-based ionic liquids, Journal of Chemical \& Engineering Data, 53(11), 2535-2539 (2008).

Khosharay, S.; K. Khosharay, K.; G. Di Nicola y M. Pierantozzi, Modelling investigation on the thermal conductivity of pure liquid, vapour, and supercritical refrigerants and their mixtures by using Heyen EOS, Physics and Chemistry of Liquids, 56(1), 124-140 (2018).

Lasdon; L.S.; A.D. Waren; A. Jain y M. Ratner, Design and testing of a generalized reduced gradient code for nonlinear programming, ACM Transactions on Mathematical Software, 4(1) 34-50 (1978).

Lawal, A.S. Prediction of vapor and liquid viscosities from the Lawal-Lake-Silberberg equation of state, 5th Symposium on Enhanced Oil Recovery, Tulsa, Estados Unidos Enero 20-23 Abril (1986).

Little, J.E. y H.T. Kennedy, A correlation of the viscosity of hydrocarbon systems with pressure, temperature and composition, Society of Petroleum Engineers Journal, 8(02), 157-162 (1968).

Marsh, K.N.; J.A. Boxall y R. Lichtenthaler, Room temperature ionic liquids and their mixtures-a review, Fluid Phase Equilibria, 219(1), 93-98 (2004).

Phillips, P., The viscosity of carbon dioxide, Proc. R. Soc., 87A, 48-52 (1912).

Poling, B.E.; J.M. Prausnitz y J.P. O'Connell, The properties of gases and liquids, Vol. 5, New York: Mcgraw-Hill (2001).

Shamshina, L.; S. Kelley; G. Gurau y R. Rogers, Chemistry: Develop ionic liquid drugs, Nature, 528(7581), 188-189, (2015).

Soave, G., Equilibrium Constants from a Modified Redlich-Kwong Equation of State, Chem. Eng. Sci., 27, 1197-1203 (1972).

Valderrama, J.O., A generalized Patel-Teja equation of state for polar and nonpolar fluids and their mixtures, J. Chem. Eng. Jpn., 23, 87-91 (1990).

Valderrama, J. O., The state of the cubic equations of state, Ind. Eng. Chem. Res., 42(8), 1603-1618 (2003).

Valderrama, J.O. y V.H. Alvarez, Correct way of reporting results when modeling supercritical phase equilibria using equations of state, Can. J. Chem. Eng., 83, 578-581 (2005).

Valderrama, J.O.; L.A. Forero y R.E. Rojas, Extension of a group contribution method to estimate the critical properties of ionic liquids of high molecular mass, Ind. Eng. Chem. Res., 54, 3480-3487 (2015).

Valderrama, J.O.; L.F. Cardona y E.R. Roberto, Correlation of lonic Liquid Viscosity using Valderrama-Patel-Teja Cubic Equation of State and the Geometric Similitude Concept. Part I: Pure lonic Liquids, Fluid Phase Equilib., en prensa (2019a).

Valderrama, J.O.; L.F. Cardona y E.R. Roberto, Correlation of lonic Liquid Viscosity using Valderrama-Patel-Teja Cubic Equation of State and the Geometric Similitude Concept. Part II: Binary Mixtures of lonic Liquids, Fluid Phase Equilib., en prensa (2019b).

Van Ness H. C.; M. M. Abbott, Classical thermodynamics of nonelectrolyte solutions, McGraw-Hill,Nueva York, USA (1982)

Wang, B.; L. Quin; T. Mu; Z. Xue y G. Gao, Are Ionic Liquids Chemically Stable?, Chemical Reviews, 117, 7113-7131 (2017). 